\title{
Efficacy, Safety, and Patient Satisfaction with a Combined Acne Treatment Regimen for the Treatment of Acne Vulgaris in Adolescent Student Athletes
}

Brian B. Adams ${ }^{1,{ }^{*}}$, Edward Lain ${ }^{2}$, JP York ${ }^{3}$ and Marie-Jose Rueda ${ }^{3}$

${ }^{1}$ Department of Dermatology, University of Cincinnati College of Medicine, Cincinnati, OH, USA

${ }^{2}$ Austin Institute for Clinical Research, Austin, TX, USA

${ }^{3}$ Galderma Laboratories, L.P., Fort Worth, TX, USA

\begin{abstract}
Background: Cleansing, medicating, moisturizing, and photoprotection comprise a complete acne vulgaris (AV) management regimen. However, complete topical AV treatment regimen investigations in athletes are scarce.

Objective: Adapalene and benzoyl peroxide (A/BPO) gel, 0.1\%/2.5\% (Epiduo ${ }^{\circledR} \mathrm{Gel}$ ) is indicated for the topical treatment of $\mathrm{AV}$ in patients 9 years of age and older. This open-label, single-center study evaluated a regimen to cleanse, medicate, moisturize, and protect the skin comprised of A/BPO gel, Cetaphil ${ }^{\circledR}$ DermaControl $^{\mathrm{TM}}$ Foam Wash and Cetaphil ${ }^{\circledR}$ DermaControl ${ }^{\mathrm{TM}}$ Moisturizer SPF 30 in student athletes (referred to as the completed Regimen).

Methods: Student athletes 12 to 18 years of age with mild to moderate acne who were actively participating in 1 of 14 Texas University Interscholastic League sanctioned athletic activities followed the completed Regimen for 8 weeks $(\mathrm{N}=28)$. Lesion counts, satisfaction, cutaneous irritation, adverse events, and adherence were assessed.

Results: After 8 weeks, lesion counts were significantly reduced compared to baseline. Good tolerability and high satisfaction were observed. Most athletes experienced no cutaneous irritation. All adverse events were mild or moderate, and the most common related adverse events were dry skin, skin exfoliation, and erythema. Most athletes exhibited exceptionally high compliance and adherence.
\end{abstract}

Conclusions: The completed Regimen is efficacious, safe, well tolerated, and helps optimize AV treatment outcomes in athletes.

Keywords: Acne vulgaris; Adapalene; Benzoyl peroxide; Athletes; Adolescents

\section{Introduction}

Acne vulgaris (AV) is a multifactorial chronic inflammatory skin condition affecting nearly all individuals between the ages of 15 and 17 and $85 \%$ between the ages of 12 and 24 [1]. AV is characterized by abnormal keratinization of the pilosebaceous epithelium, increased sebum production, Propionibacterium acnes proliferation, and inflammation. The long term dermatologic consequences of AV can adversely affect quality of life, self-esteem, and social relationships. These negative effects can be alleviated by early implementation of an effective treatment regimen [2].

Athletes may be especially vulnerable to $\mathrm{AV}$ due to increased sweating, contact with sporting equipment, and use of sunscreens that promote the blockage of skin pores (comedogenic) [3-6]. Prevention is a primary recommendation; however, it can be difficult for student athletes to maintain a daily skincare regimen due to academic and athletic schedules. Prompt treatment minimizes disruptions in athletic participation and long term residual effects (such as scarring) [4]. Adolescents are particularly vulnerable to the negative psychological consequences of AV [7-11], but few adolescents seek AV treatment [12]. Poor skin health education and misinformation may contribute to the reluctance to seek treatment. Athletes, in particular, may avoid even topical therapies as both they and their clinicians may perceive high potential for side effects that could deleteriously affect activity and performance. AV treatment adherence is frequently low [1316], and insufficient physician evaluated or patient perceived clinical improvement and overly complex treatment regimens contribute to non-adherence [13-16]. In contrast, increased initial disease severity, clinical improvement, satisfaction, and education were associated with increased adherence.
Because systemic AV treatments, such as oral antibiotics and isotretinoin, have been associated with increased risk of injury, topical therapy should be the first choice in the athlete [17-19]. Unfortunately, topical therapies are frequently associated with tolerability reactions [20,21]. Therefore, an ideal treatment regimen must: 1) Cleanse without irritating the skin or removing the constitutive lipids that help maintain the epidermal barrier and skin hydration; 2) Medicate, reducing lesion counts without inducing excessive tolerability reactions; 3) Moisturize, alleviating irritation and protecting the skin from water loss without promoting comedone formation; and 4) Protect the skin from ultraviolet light exposure, especially the skin of individuals photosensitized by AV treatments [22-26].

A daily AV management regimen comprised of adapalene and benzoyl peroxide (A/BPO) gel, $0.1 \% / 2.5 \%$ (Epiduo ${ }^{\circ} \mathrm{Gel}$ ), Cetaphil DermaControl $^{\mathrm{Tm}}$ Foam Wash, and Cetaphil ${ }^{\circ}$ DermaControl ${ }^{\mathrm{Tm}}$ Moisturizer SPF 30 (referred to as the CoMMPlete Regimen) was designed to synergistically support AV management, provide strong photoprotection, and promote the natural structure and function

*Corresponding author: Brian B. Adams, Department of Dermatology, University of Cincinnati College of Medicine, Cincinnati, OH, USA, Tel: 513-558-1903; Fax 513-584-3531; E-mail: adamsbb@ucmail.uc.edu

Received January 14, 2016; Accepted January 28, 2016; Published February 05, 2016

Citation: Adams BB, Lain E, York JP, Rueda MJ (2016) Efficacy, Safety, and Patient Satisfaction with a Combined Acne Treatment Regimen for the Treatment of Acne Vulgaris in Adolescent Student Athletes. J Gen Pract 4: 228. doi: 10.4172/23299126.1000228

Copyright: @ 2016 Adams BB, et al. This is an open-access article distributed under the terms of the Creative Commons Attribution License, which permits unrestricted use, distribution, and reproduction in any medium, provided the original author and source are credited. 
of the epidermal barrier $[21,22,24,27,28]$. A/BPO gel is a fixed dose combination of adapalene $0.1 \%$ and benzoyl peroxide $2.5 \%$ that provides significantly greater AV treatment efficacy than either product alone, with a similar safety profile to adapalene monotherapy [29-32]. A subgroup analysis of $3 \mathrm{~A} / \mathrm{BPO}$ studies in adolescents between 12 and 17 years of age confirmed that $\mathrm{A} / \mathrm{BPO}$ gel $0.1 \% / 2.5 \%$ had superior efficacy to either monotherapy [32]. The cleansing component, Cetaphil ${ }^{\circ}$ DermaControl $^{\mathrm{TM}}$ Foam Wash, is a mild and nonirritating foaming skin cleanser specifically designed for acne prone skin and previously demonstrated to be safe and well-tolerated $[21,33]$. The moisturizing and sunscreen component, Cetaphil ${ }^{\circ}$ DermaControl ${ }^{\mathrm{TM}}$ Moisturizer SPF 30, has also been demonstrated to be well-tolerated and noncomedogenic $[21,33]$.

We investigated this 3-component regimen designed to provide nonirritating gentle skin cleansing, support a healthy epidermal barrier, and provide UV protection among athletes. We report the efficacy, safety, subject satisfaction, and adherence in an open-label, singlecenter study of this target population.

\section{Methods}

\section{Study design}

This was an 8-week, open-label, multi-center study of an AV treatment regimen for adolescent athletes with a clinical diagnosis of mild or moderate acne that examined changes in lesion counts, subject satisfaction, and adherence. Athletes were treated with commercially packaged A/BPO gel in conjunction with Cetaphil ${ }^{\circ}$ DermaControl ${ }^{\text {TM }}$ Foam Wash and Cetaphil ${ }^{\circ}$ DermaControl ${ }^{\text {Ts }}$ Moisturizer SPF 30 (all products were supplied by Galderma Laboratories L.P., Fort Worth, $\mathrm{TX}$ ). Athletes were instructed to use the A/BPO gel (once daily) in conjunction with the foam wash (at least twice daily) and moisturizer with sunscreen (once daily and additionally 15 minutes prior to participation in outdoor sports if more than 2 hours elapsed since morning application). Every attempt was made to keep any allowed concomitant therapy dosing and regimen constant during the study. The first subject was enrolled on August 20, 2014, and the last subject complete d the study on December 21, 2014.

\section{Subject eligibility}

Enrolled athletes were aged 12 to 18 and actively participating in 1 of 14 Texas University Interscholastic League sanctioned athletic activities (Baseball, Basketball, Cross Country, Football, Golf, Soccer, Softball, Swimming, Diving, Team Tennis, Tennis, Track and Field, Volleyball, and Wrestling). Eligible athletes were included if they were diagnosed with $\mathrm{AV}$, deemed eligible for $\mathrm{A} / \mathrm{BPO}$ treatment, had a minimum of 20 but not more than 50 inflammatory (papules and pustules) facial lesions (excluding the nose) and had a minimum of 30 but not more than 100 noninflammatory (open comedones and closed comedones) facial lesions (excluding the nose). Girls were required to have a negative urine pregnancy test at the baseline clinic visit and agreed to practice 1 effective nonhormonal method of contraception for the study duration. Acceptable contraception methods included abstinence, IUD (inserted 30 days prior to baseline), double barrier method, bilateral tubal ligation, or vasectomized partner (at least 90 days prior to baseline).

Athletes were not eligible if they met any of the following criteria: the presence of nodules or cysts; pregnant, nursing, or planning a pregnancy during the study; any visible skin condition or facial hair that would interfere with the study evaluations; the presence of any risk factors or allergies to any of the ingredients described in the precautions, warnings, and contraindications of the study drug's package insert; any systemic or dermatological disorder which could increase the rate of adverse reactions, or interfere with the conduct of the study or interpretation of results; the use, or planned use, during the study period of any systemic or topical acne medication, or other medications or procedures that could interfere with the test results.

\section{Ethical conduct}

This study was conducted in accordance with ICH and Good Clinical Practice principles, the FDA Code of Federal Regulations, and federal and local regulatory requirements. Before study initiation, the protocol and applicable study documents were reviewed and approved by a central institutional review board (Schulman Associates IRB, Inc., Cincinnati, OH). Subject assent and parent or legal guardian consent was obtained for all subjects under 18 years of age. For subjects who were 18 years of age, consent was obtained from the subjects themselves. Before inclusion in the study or participation in any study procedures, athletes aged 12 to 17 signed the assent form and their parent signed the ICF. Eighteen year-old athletes signed ICF themselves.

\section{Subject evaluation}

Up to 5 scheduled visits occurred over the course of the study: screening, visit 1 (baseline), visit 2 (week 2), visit 3 (week 4), and visit 4 (week 8, study endpoint). AV lesion counts, cutaneous irritation assessments, photographs, adverse event recording and compliance assessments were completed at all study visits. AV lesion counts included evaluation of facial inflammatory (papules, pustules) and noninflammatory (open and closed comedones) lesions. The baseline and screening visit could occur on the same day. Athletes were evaluated at screening and 4 scheduled study visits. During the screening visit, topics relating to the conduct of the study were explained to each subject, subject demographic and medical history information were collected, and lesion counts were performed. Athletes completed a baseline questionnaire at visit 1 and end of study satisfaction and adherence questionnaires at visit 4 .

\section{End of study satisfaction questionnaire}

The 15-question end of study subject satisfaction questionnaire was verbally administered by study staff. For athletes less than 16 years old, the questionnaire was administered to the subject in the presence of a parent or legal guardian who could also answer the questions. The recorded response was the best response based on the combined parent/subject feedback.

\section{Adherence questionnaire}

The end of study, 3-item adherence questionnaire was verbally administered by study staff. A parent or legal guardian was present for athletes less than 16 years old. Parent and subject feedback was combined, and a single best response was recorded.

\section{Safety Measurements}

Safety assessments were performed for all athletes throughout the course of the study, and all subject or investigator reported adverse events (AEs) were recorded at each study visit. Cutaneous irritation assessments of erythema, scaling, dryness, and stinging/burning were assessed by a board-certified dermatologist on a 4-point scale (none, mild, moderate, and severe) at baseline and each post-baseline visit.

\section{Other assessments}


Treatment compliance for the study treatment regimen was based on questioning the subject at each visit and collecting study product weights prior to dispensation and at end of study. Athletes were photographed at visits 1 through 4/exit.

\section{Statistical analysis}

For all efficacy variables, descriptive statistics were computed based on the nature of the variable (continuous or categorical). Categorical variables were summarized by frequency and percentage for each response category $(\mathrm{N}, \%)$. Continuous variables were summarized using means, medians, minimum, maximum, and standard deviations for the data collected at each visit and the changes and percent changes from baseline at each post baseline visit when appropriate. Last observation carried forward was used to impute missing efficacy data. The change and percent change from baseline in lesion counts was analyzed by $t$ tests and signed rank tests for the mean and median, respectively.

Safety endpoints included adverse events and cutaneous irritation assessments. No statistical testing was conducted on the safety parameters.

\section{Results}

\section{Demographics and disposition}

Twenty-eight athletes were enrolled and 25 completed the study. One athlete withdrew due to an adverse event, 1 requested to be discontinued, and 1 was lost to follow up. All 28 athletes were included in the safety and intent-to-treat populations. One or more protocol deviations were recorded for 12 athletes during the study, including 1 athlete with an exclusion violation, 3 athletes who were dosing noncompliant and 9 athletes who had off-schedule visits. Subject disposition is depicted in Table 1.

Most enrolled athletes were men (78.6\%), White (82.1\%), and nonHispanic or Latino (71.4\%). Athletes ranged from 12 to 18 years of age, with a mean age of 15.0 years. Most athletes had Fitzpatrick Skin Types II-IV (82.2\%). Study demographics and baseline characteristics of enrolled athletes are depicted in Table 2.

Football (39.3\%), soccer (25.0\%), baseball (17.9\%), basketball (17.9\%), and track and field (14.3\%) were the most common sports (Figure 1). Sporting equipment that covered all or part of the face was reported as worn by $57.1 \%$ of athletes, and $89.3 \%$ of athletes reported that they participated in outdoor sports.

\section{Lesion counts}

The mean total lesion count at baseline was 90.5 (range, 62 to 128 lesions), with a mean of 32.8 inflammatory lesions (range, 21 to 47 inflammatory lesions) and a mean of 57.6 non-inflammatory lesions (range, 34 to 97 non-inflammatory lesions) (Table 3). Lesion count reduction was observed as early as week 2 , progressing to a significant reduction in total $(29.6 \%)$, inflammatory $(35.7 \%)$, and non-inflammatory (27.1\%) lesion counts at week $8(P<0.001)$ (Figures 2 and 3$)$.

\section{Subject satisfaction questionnaire results}

Most athletes agreed or strongly agreed with all questions in the end of study subject satisfaction questionnaire, including overall satisfaction with the regimen (Figure $4 \mathrm{~A}$ and $4 \mathrm{~B}$ ). The athletes felt that the regimen was easy to use and reported a positive difference in skin appearance and acne.

A notable shift in subject attitudes was observed at study exit

\begin{tabular}{|c|c|}
\hline Disposition & Athletes, $\mathbf{n}$ (\%) \\
\hline Enrolled & 28 \\
\hline ITT population & $28(100)$ \\
\hline Safety population & $28(100)$ \\
\hline Discontinued & $3(10.7)$ \\
\hline Subject's request & $1(3.6)$ \\
\hline Adverse event & $1(3.6)$ \\
\hline Lost to follow-up & $1(3.6)$ \\
\hline CoMMPlete d & $25(89.3)$ \\
\hline Athletes with protocol deviations & $12(42.9)$ \\
\hline Inclusion/exclusion violation & $1(3.6)$ \\
\hline Off-schedule visit(s) & $9(32.1)$ \\
\hline Not dosing compliant & $3(10.7)$ \\
\hline
\end{tabular}

Table 1: Disposition of athletes.

\begin{tabular}{|c|c|c|}
\hline Demographic Variable & Measure & Athletes \\
\hline \multirow{3}{*}{ Age, y } & Mean (SD) & $15.0(1.4)$ \\
\hline & Median & 15 \\
\hline & Range (min, max) & $(12.0,18.0)$ \\
\hline \multirow{2}{*}{ Gender, n (\%) } & Male & $22(78.6)$ \\
\hline & Female & $6(21.4)$ \\
\hline \multirow[b]{2}{*}{ Ethnicity, n (\%) } & Hispanic or Latino & $8(28.6)$ \\
\hline & $\begin{array}{l}\text { Not Hispanic or } \\
\text { Latino }\end{array}$ & $20(71.4)$ \\
\hline \multirow[b]{2}{*}{ Race, n (\%) } & White & $23(82.1)$ \\
\hline & $\begin{array}{l}\text { Black or African } \\
\text { American }\end{array}$ & $5(17.9)$ \\
\hline \multirow{3}{*}{ Skin Type, n (\%) } & Dry & $4(14.3)$ \\
\hline & Normal & $14(50.0)$ \\
\hline & Oily & $10(35.7)$ \\
\hline \multirow{6}{*}{ Fitzpatrick Skin Type, n (\%) } & I & 0 \\
\hline & II & $10(35.7)$ \\
\hline & III & $8(28.6)$ \\
\hline & IV & $5(17.9)$ \\
\hline & V & 0 \\
\hline & VI & $5(17.9)$ \\
\hline \multirow{3}{*}{ History of acne, $y$} & Mean (SD) & $3.1(1.4)$ \\
\hline & Median & 2.8 \\
\hline & Range (min, max) & $(0.3,6.7)$ \\
\hline
\end{tabular}

Table 2: Athlete demographics.

regarding the acne specific moisturizer and sunscreen elements of the regimen (Figure 4B). At baseline, only $10.7 \%$ of athletes indicated that they had used any acne specific facial moisturizer within 30 days and $78.6 \%$ reported no facial moisturizer use at all. At baseline, only $28.6 \%$ of athletes felt that it was important or very important to use a facial moisturizer with sunscreen (Figure 4C). Furthermore, only $17.8 \%$ of athletes thought it was important or very important to apply sunscreen before engaging in sports activities at the baseline visit, and $85.7 \%$ responded that they did not regularly apply sunscreen before going outdoors. However, at study exit, more than $60 \%$ agreed or strongly agreed that an acne specific SPF moisturizer was personally important (Figure 4D). The majority of athletes at study exit responded that they would recommend the regimen to others, including others in their sport, and that they would continue using the regimen themselves.

\section{Compliance and adherence assessment}

As evaluated by the study questionnaires and post study product weights, most athletes demonstrated remarkably good adherence and compliance to the study protocol and regimen. Athletes were $89.3 \%$ 
Citation: Adams BB, Lain E, York JP, Rueda MJ (2016) Efficacy, Safety, and Patient Satisfaction with a Combined Acne Treatment Regimen for the Treatment of Acne Vulgaris in Adolescent Student Athletes. J Gen Pract 4: 228. doi: 10.4172/2329-9126.1000228

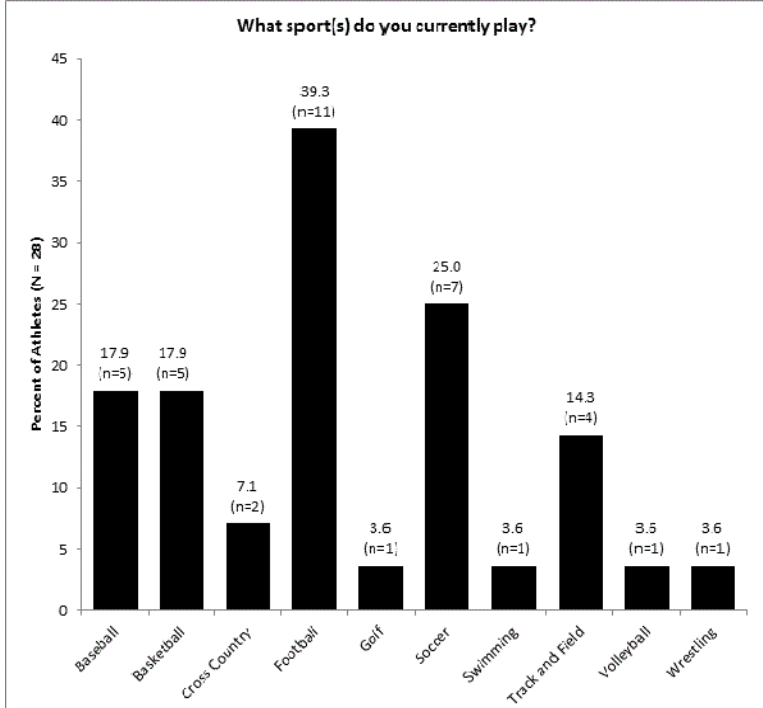

Figure 1: Sports subgroup analysis. Athletes were asked what sport(s) they currently played. Simultaneous participation in more than 1 sport was permitted.

\begin{tabular}{|l|l|}
\hline Disease Characteristic & Lesion Count \\
\hline Total Lesions & \\
\hline Mean (SD) & $90.5(15.9)$ \\
\hline Median & 91 \\
\hline Range (min, max) & $(62.0,128.0)$ \\
\hline Inflammatory Lesions & \\
\hline Mean (SD) & $32.8(6.9)$ \\
\hline Median & 32 \\
\hline Range (min, max) & $(21.0,47.0)$ \\
\hline Noninflammatory Lesions & \\
\hline Mean (SD) & $57.6(13.9)$ \\
\hline Median & 55 \\
\hline Range (min, max) & $(34.0,97.0)$ \\
\hline
\end{tabular}

Table 3: Baseline disease characteristics.

dosing compliant with the overall CoMMPlete regimen, and only 3 athletes were found to be non-dosing compliant. Athletes were $100 \%$ compliant with foam wash usage, $100 \%$ compliant with SPF moisturizer usage, $96.4 \%$ compliant with SPF moisturizer reapplication, and $92.9 \%$ compliant with $\mathrm{A} / \mathrm{BPO}$ gel usage. Adherence questionnaire results were similarly favorable. Most athletes were able to correctly identify all study products, and over $90 \%$ were able to correctly identify the correct prescription product by the brand name (Epiduo Gel). Over $90 \%$ reported using A/BPO gel and SPF moisturizer at least once per day as directed, and over $85 \%$ used the facial wash at least twice per day as directed.

\section{Tolerability}

The CoMMPlete regimen was safe and well tolerated by participants. Most athletes reported no scaling, dryness, or stinging and burning. Eighteen athletes experienced $31 \mathrm{AEs}$, most of which were mild in severity. Nineteen AEs were considered related to regimen use, including erythema, dry skin, skin exfoliation, skin burning sensation, application site pain, and sunburn (Table 4). One subject discontinued the regimen due to an $\mathrm{AE}$ that was considered related to the regimen (skin burning sensation). This AE resolved with no residual effects

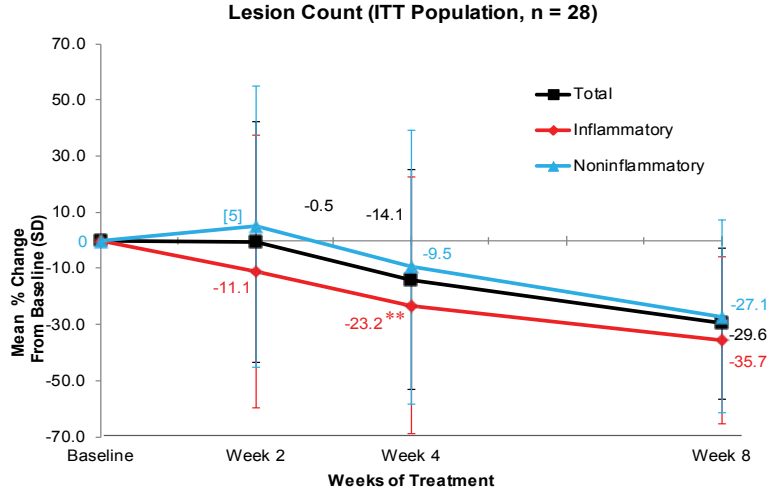

Figure 2: Mean percent change from baseline in lesion counts. ${ }^{*} P<0.001$ and ${ }^{* *} P<0.05$ compared to baseline. ITT, intent to treat, $\mathrm{n}=28$. SD, standard deviation. $P$ values were calculated using 2 sided paired $t$ tests.
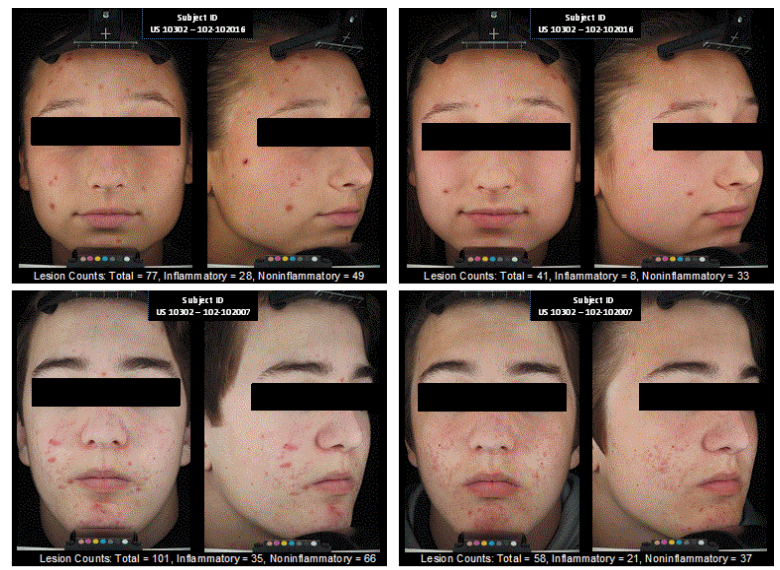

Figure 3: Clinical photographs. Representative photographs of adolescent student athletes. A) 15 year old White Hispanic girl (Fitzpatrick skin type IV, normal skin, 2.72 year acne history), B) 15 year old White boy (Fitzpatrick skin type III, dry skin, 2.66 year acne history). Photographs show athletes at the baseline and week 8 visits.

after discontinuation. No serious AEs were reported during the study. A slight increase in athletes experiencing mild to moderate erythema, scaling, dryness, and stinging and burning was reported by investigators at week 2 . By week 8 , scaling, dryness, and stinging and burning were similar to baseline (Figure 5).

\section{Discussion}

A successful AV treatment regimen must effectively cleanse, medicate, moisturize, and protect the skin; however, subject satisfaction and adherence are equally important determinants of treatment success. Athletes face a number of specific skin care challenges that may increase the difficulty of treating AV, including extensive sunlight, heat, physical contact between participants, and occlusive and abrasive equipment. These factors may be the reason why the baseline lesion counts were higher in the athletes enrolled in the current study than in past investigations of non-targeted subjects [27]. Therefore, the selection of a gentle skin care regimen that incorporates a sunscreen component is especially important for successful AV treatment in athletes. This report describes the successful treatment of $\mathrm{AV}$ in athletes 
A

Basline questionnaire responses

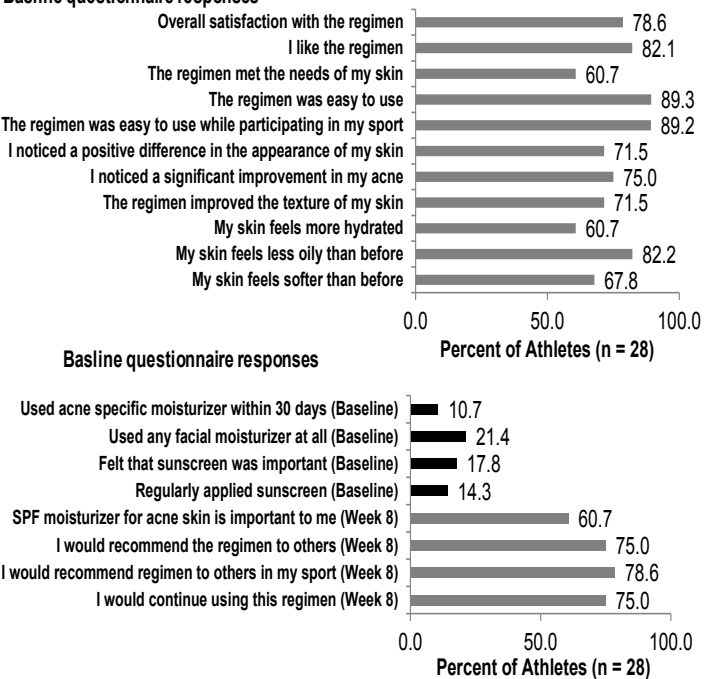

Baseline: How important is it for you to use a facial moisturizer with sunscreen?

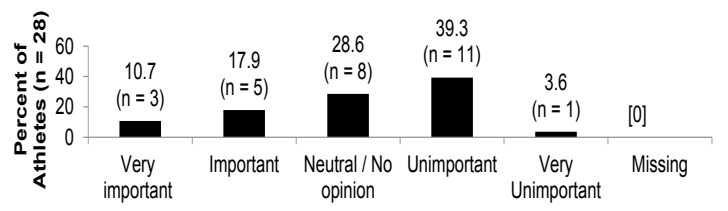

Exit: Moisturizer with sunscreen made for acne prone skin is important to me.

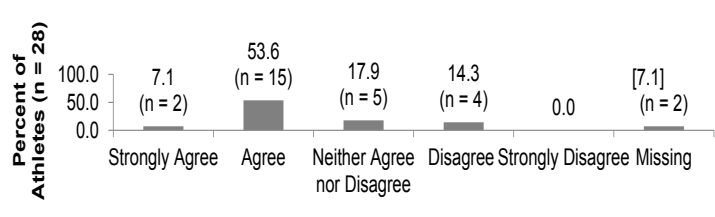

Figure 4: Subject satisfaction and skin care attitudes.

using the CoMMPlete regimen. This safe and well-tolerated regimen significantly reduced end of study total lesion counts, and athletes using the regimen exhibited exceptional satisfaction and adherence.

When compared with a population of non-athletes from a similar previous investigation, the athletes in the current study were much less likely to feel that a facial moisturizer with sunscreen was an important part of their daily self-care routine at baseline $(17.8 \%$ current $v s$. $34.2 \%$ previous) [27]. Most participants indicated that they had used no sunscreen, acne specific face wash, or moisturizer within 30 days (Figure 3). Furthermore, the athletes placed a lower priority on skin care despite exhibiting higher baseline mean total lesion counts than was seen in the similar previous study (90.5 vs. 76.7) [27].

While the exit questionnaire responses indicated that athletes placed less importance on moisturizer with sunscreen $(60.7 \%$ current vs. $70.2 \%$ previous), the more than three-fold difference between the baseline and exit attitudes of the student athletes suggests that the CoMMPlete regimen had a meaningful positive impact on perceived quality of life. Most athletes agreed or strongly agreed with all end of study satisfaction questions. The remarkable shift in subject attitudes strongly suggests that the baseline apathy of the athletes may have been at least partially attributable to a lack of skin care knowledge. The end of study results indicate that this population actually cares very strongly about the health and appearance of their skin if they are given access to an easy to use and well-tolerated skin care regimen.

Underscoring the positive change in attitudes, the student athletes exhibited exceptional adherence to the foam wash and SPF moisturizer, and subject satisfaction and adherence in the current study were better than is typically seen for topical AV therapies [13]. Compliance ranged between 90 and $100 \%$ for each component of the regimen, and the majority of the athletes reported excellent adherence in the exit questionnaire. Satisfaction may be among the most important predictors of adherence in global assessments of AV therapy, and most athletes reported a perceived improvement in acne and the general health and appearance of their skin. Additionally, athletes reported that the regimen was a good lifestyle fit, and that the treatments were easy to use [13]. Furthermore, the majority of athletes responded that they intended to continue using the regimen and would recommend it to their peers. These results suggest that increased education and access to an easy to use, nonirritating regimen could contribute to meaningful skin health and AV treatment improvements for student athletes.

This was a single-center, single-arm, open-label investigation with a relatively small sample size. Because the athletes enrolled in this study chose to participate in this investigation, they may have had a higher 
Citation: Adams BB, Lain E, York JP, Rueda MJ (2016) Efficacy, Safety, and Patient Satisfaction with a Combined Acne Treatment Regimen for the Treatment of Acne Vulgaris in Adolescent Student Athletes. J Gen Pract 4: 228. doi: 10.4172/2329-9126.1000228

Page 6 of 7

\begin{tabular}{|c|c|c|}
\hline & Athletes, $\mathrm{n}(\%)$ & Events \\
\hline Total events & $18(64.3)$ & 31 \\
\hline Related events & $12(42.8)$ & 19 \\
\hline \multicolumn{3}{|c|}{ Related events by system organ class } \\
\hline \multicolumn{3}{|c|}{ Skin and subcutaneous tissue disorders } \\
\hline Erythema & $4(14.3)$ & 4 \\
\hline Dry skin & $7(25.0)$ & 7 \\
\hline Skin exfoliation & $5(17.9)$ & 5 \\
\hline Skin burning sensation & $1(3.6)$ & 1 \\
\hline \multicolumn{3}{|c|}{ Injury, poisoning and procedural complications } \\
\hline Sunburn & $1(3.6)$ & 1 \\
\hline \multicolumn{3}{|c|}{ General disorders and administration site conditions } \\
\hline Application site pain & $1(3.6)$ & 1 \\
\hline
\end{tabular}

Table 4: All related adverse events.

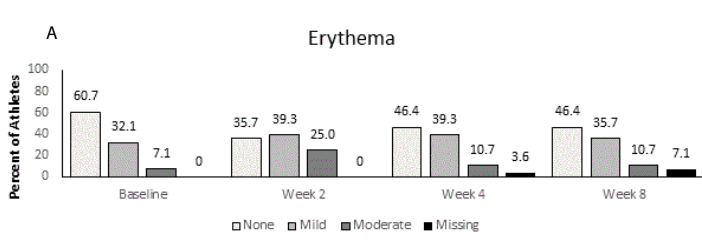

B

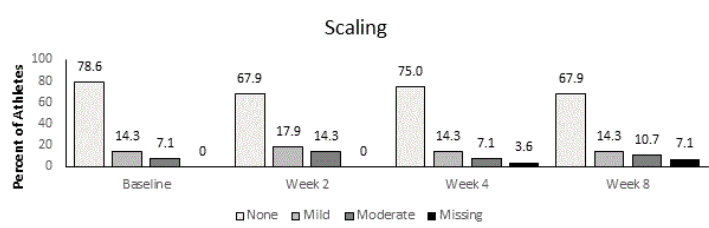

Dryness
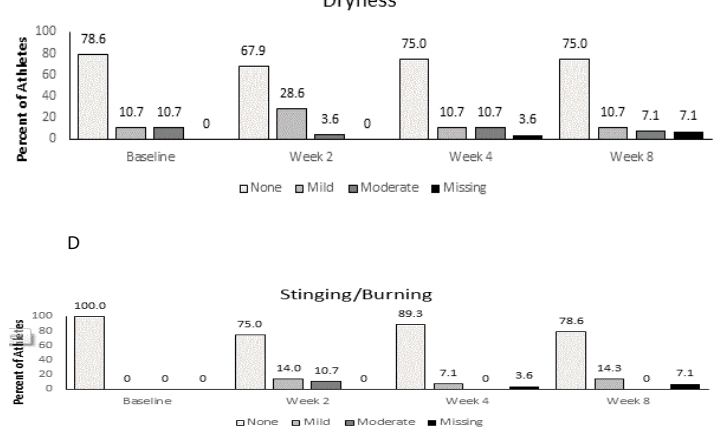

E

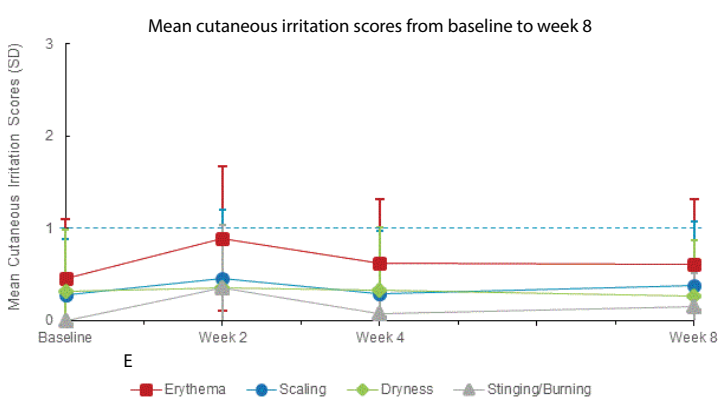

Figure 5: Cutaneous irritation assessment. Cutaneous irritation assessments of A) erythema, B) Scaling, C) Dryness, D) Stinging/burning were conducted at the baseline through week 8 visits, E) Mean cutaneous irritation scores from baseline to week 8 . Safety population, $n=28$. 4-point scale $(0=n o n e$, $1=$ mild, $2=$ moderate, and $3=$ severe). level of motivation and study adherence than would be seen in the general population. Most athletes enrolled in this study were men, and future investigations should attempt to recruit a higher proportion of women. Future studies should also attempt to widen the distribution and variety of sports included in the investigation, and these studies should be conducted with larger sample sizes recruited from multiple geographically distinct populations of varying ages.

In conclusion, the CoMMPlete Regimen may be an excellent $\mathrm{AV}$ treatment regimen for athletes aged 12 to 18 . Athletes exhibited significant end of study improvement in total lesion counts, a very low incidence of adverse effects, and unusually high levels of adherence and satisfaction.

\section{Acknowledgements}

Funding: Galderma Laboratories, L.P. funded this study.

\section{Conflicts of Interest and Source of Funding}

Dr. Lain received investigator fees to conduct the study. Dr. Adams is a Galderma Laboratories, L.P. paid consultant. Drs. Rueda and York are employees of Galderma Laboratories, L.P.

\section{References}

1. Bhate K, Williams HC (2013) Epidemiology of acne vulgaris. Br J Dermatol 168: 474-485.

2. Zaghloul SS, Cunliffe WJ, Goodfield MJ (2005) Objective assessment of compliance with treatments in acne. Br J Dermatol 152: 1015-1021.

3. (2012-2013) National Federation of State High School Associations (NFHS) High School Athletics Participation Survey [The National Federation of State High School Associations web site]

4. Adams BB (2010) Sports dermatoses: perils of occlusion for the athlete. Pract Dermatol. AL008-AL009.

5. Adams BB (2001) Sports dermatology. Dermatol Nurs 13: 347-348, 351-8, 363.

6. Adams BB (2006) Sports Dermatology. New York, NY: Springer.

7. Uslu G, Sendur N, Uslu M, Savk E, Karaman G, et al. (2008) Acne: prevalence perceptions and effects on psychological health among adolescents in Aydin, Turkey. J Eur Acad Dermatol Venereol 22: 462-469.

8. Halvorsen JA, Stern RS, Dalgard F, Thoresen M, Bjertness E, et al. (2011) Suicidal ideation, mental health problems, and social impairment are increased in adolescents with acne: a population-based study. J Invest Dermatol 131: 363-370.

9. Isaacsson VC, Almeida HL Jr, Duquia RP, Breunig Jde A, Souza PR (2014) Dissatisfaction and acne vulgaris in male adolescents and associated factors. An Bras Dermatol 89: 576-579.

10. Poli F, Auffret N, Beylot C, Chivot M, Faure M, et al. (2011) Acne as seen by adolescents: results of questionnaire study in 852 French individuals. Acta Derm Venereol 91: 531-536. 
Citation: Adams BB, Lain E, York JP, Rueda MJ (2016) Efficacy, Safety, and Patient Satisfaction with a Combined Acne Treatment Regimen for the Treatment of Acne Vulgaris in Adolescent Student Athletes. J Gen Pract 4: 228. doi: 10.4172/2329-9126.1000228

11. Chen CL, Kuppermann M, Caughey AB, Zane LT (2008) A community-based study of acne-related health preferences in adolescents. Arch Dermatol 144: 988-994.

12. Nijsten T, Rombouts S, Lambert $\mathrm{J}$ (2007) Acne is prevalent but use of its treatments is infrequent among adolescents from the general population. J Eur Acad Dermato Venereol 21: 163-168.

13. Dréno B, Thiboutot D, Gollnick H, Finlay AY, Layton A, et al. (2010) Large-scale worldwide observational study of adherence with acne therapy. Int J Dermatol 49 : 448-456.

14. Flanders PA, McNamara JR (1985) Enhancing acne medication compliance: a comparison of strategies. Behav Res Ther 23: 225-227.

15. Lee IA, Maibach HI (2006) Pharmionics in dermatology: a review of topical medication adherence. Am J Clin Dermatol 7: 231-236.

16. Jones-Caballero M, Pedrosa E, Peñas PF (2008) Self-reported adherence to treatment and quality of life in mild to moderate acne. Dermatology 217: 309-314.

17. Koch PE, Ryder HF, Dziura J, Njike V, Antaya RJ (2008) Educating adolescents about acne vulgaris: a comparison of written handouts with audiovisual computerized presentations. Arch Dermatol 144: 208-214.

18. Fayock K, Voltz M, Sandella B, Close J, Lunser M, et al. (2014) Antibiotic precautions in athletes. Sports Health 6: 321-325.

19. Pavlis MB, Lieblich $L$ (2013) Isotretinoin-induced skin fragility in a teenaged athlete: a case report. Cutis 92 : 33-34.

20. Thiboutot D, Gollnick H, Bettoli V, Dréno B, Kang S, et al. (2009) New insights into the management of acne: an update from the Global Alliance to Improve Outcomes in Acne group. J Am Acad Dermatol 60: S1-50.

21. Del Rosso JQ, Brandt S (2013) The Role of Skin Care as an Integral Component in the Management of Acne Vulgaris: Part 2: Tolerability and Performance of a Designated Skin Care Regimen Using a Foam Wash and Moisturizer SPF 30 in Patients with Acne Vulgaris Undergoing Active Treatment. J Clin Aesthet Dermato 6: 28-36.

22. Goodman G (2009) Cleansing and moisturizing in acne patients. Am J Clin Dermatol 10 Suppl 1: 1-6.
23. Subramanyan K (2004) Role of mild cleansing in the management of patient skin Dermatol Ther 17 Suppl 1: 26-34

24. Del Rosso JQ (2013) The role of skin care as an integral component in the management of acne vulgaris: part 1: the importance of cleanser and moisturizer ingredients, design, and product selection. J Clin Aesthet Dermatol 6: 19-27.

25. Yamamoto A, Takenouchi K, Ito M (1995) Impaired water barrier function in acne vulgaris. Arch Dermatol Res 287: 214-218.

26. Bowe WP, Kircik LH (2014) The importance of photoprotection and moisturization in treating acne vulgaris. J Drugs Dermatol 13: s89-94.

27. Del Rosso JQ, Gold M, Rueda MJ, Brandt S, Winkelman WJ (2015) Efficacy, safety and subject satisfaction of a specified skin care regimen to cleanse, medicate, moisturize, and protect the skin of patients under treatment for acne vulgaris. J Clin Aesthet Dermatol 8: 22-30.

28. Jacobs A, Starke G, Rosumeck S, Nast A (2014) Systematic review on the rapidity of the onset of action of topical treatments in the therapy of mild-to-moderate acne vulgaris. Br J Dermatol 170: 557-564.

29. Thiboutot DM, Weiss J, Bucko A, Eichenfield L, Jones T, et al. (2007) Adapalenebenzoyl peroxide, a fixed-dose combination for the treatment of acne vulgaris: results of a multicenter, randomized double-blind, controlled study. J Am Acad Dermatol 57: 791-799.

30. Gold LS, Tan J, Cruz-Santana A, Papp K, Poulin Y, et al. (2009) A North American study of adapalene-benzoyl peroxide combination gel in the treatment of acne. Cutis 84: 110-116.

31. Gollnick HP, Draelos Z, Glenn MJ, Rosoph LA, Kaszuba A, et al. (2009) Adapalenebenzoyl peroxide, a unique fixed-dose combination topical gel for the treatment of acne vulgaris: a transatlantic, randomized, double-blind, controlled study in 1670 patients. Br J Dermatol 161: 1180-1189.

32. Eichenfield LE, Jorizzo JL, Dirschka T, Taub AF, Lynde C, et al. (2010) Treatment of 2,453 acne vulgaris patients aged 12-17 years with the fixed-dose adapalenebenzoyl peroxide combination topical gel: efficacy and safety. J Drugs Dermatol 9: 1395-1401.

33. Hensley D, Meckfessel MH (2015) Tolerability of a Skin Care Regimen Formulated for Acne-Prone Skin in Children. Pediatr Dermatol 32: 501-505. 